\title{
Stimulatory effect of indole-3-acetic acid and continuous illumination on the growth of Parachlorella kessleri**
}

\author{
Edyta Magierek, Izabela Krzemińska*, and Jerzy Tys \\ Institute of Agrophysics, Polish Academy of Sciences, Doświadczalna 4, 20-290 Lublin, Poland
}

Received January 10, 2017; accepted July 6, 2017

\begin{abstract}
A b s t r a c t. The effects of the phytohormone indole-3-acetic acid and various conditions of illumination on the growth of Parachlorella kessleri were investigated. Two variants of illumination: continuous and photoperiod $16 / 8 \mathrm{~h}$ (light/dark) and two concentrations of the phytohormone $-10^{-4} \mathrm{M}$ and $10^{-5} \mathrm{M}$ of indole3 -acetic acid were used in the experiment. The results of this study show that the addition of the higher concentration of indole-3-acetic acid stimulated the growth of $P$. kessleri more efficiently than the addition of the lower concentration of indole-3-acetic acid. This dependence can be observed in both variants of illumination. Increased biomass productivity was observed in the photoperiod conditions. Both the addition of the phytohormone and the conditions of the illumination had an impact on the number of $P$. kessleri cells. An increased number of cells was observed under the conditions of continuous illumination. This result has shown that the continuous illumination and the higher concentration of the phytohormone stimulated the growth of P. kessleri more effectively than the shorter duration of light (16/8 h (light/dark)).

K e y w o r d s: light, photoperiod, microalgae, indole-3-acetic acid, Parachlorella kessleri
\end{abstract}

\section{INTRODUCTION}

In recent years, there has been growing interest in the biomass of unicellular algae as an alternative energy source. Microalgae, specifically those included in the group of green algae, can be used in production of the third generation of biofuels: biodiesel, biohydrogen, and biomethanol. Algal biomass can also be used in nutrition as food supplements, which are a source of many valuable substances as well as micro- and macronutrients, in the pharmaceutical industry as nutraceuticals, and in cosmetic industry as cosmeceuticals (Mata et al., 2010).

*Corresponding author e-mail: i.krzeminska@ipan.lublin.pl

**This work was partly financed by the National Science Centre, Poland; project No. 2016/23/D/NZ9/02670 (2017-2020).
Despite such wide possibilities of using algal biomass, commercialization of biomass production is still a challenge due to the high costs. An increase in the efficiency of production of biomass and valuable intracellular metabolites can improve the profitability of algal cultivation. Therefore, it is important to understand better the factors influencing the growth of microalgae. The main factors that exert an effect on the growth of algae include light, access to nutrients, temperature, $\mathrm{pH}$, salinity, environmental stress, as well as addition of other growth-promoting substances, such as plant hormones (Markou et al., 2013; Salama et al., 2014).

Plant hormones, also called phytohormones, are signal molecules that induce a reaction in the plant organism already at trace concentrations. The main phytohormones e.g. gibberellins, auxins, cytokinins (CKs), abscisic acid, ethylene, and brassinosteroids (BRs) are plant growth and development regulators. The most common and best explored auxin is indole-3-acetic acid (IAA) ( $\mathrm{Lu}$ and $\mathrm{Xu}, 2015)$.

Phytohormones are also found in algae at comparable concentrations as those in terrestrial plants. The greatest number of plant hormones has been detected in Chlorophyta (green algae). IAA has been detected in several unicellular and multicellularalgal species, i.e. Chlorella, Enteromorpha, Cladophora, Caulerpa (Chlorophyta) Fucus, Laminaria, Porphyra, Botryocladia (Rhodophyta), Ascophyllum, Macrocystis (Phaeophyta), and several others. The spectrum of biological activities of algal hormones

(C) 2017 Institute of Agrophysics, Polish Academy of Sciences 
partly overlaps with that in terrestrial plants; however, it is being discussed whether the function of the algal hormonal system is the same as in terrestrial plants (Lau et al., 2009).

There are reports in the literature that the addition of phytohormones, particularly auxins, to microalgal cultures can increase the productivity of biomass as well as the number of cells and the content of metabolites in microalgal biomass. Exogenous auxin increases the growth rate of algae such as Chlorella vulgaris, Chlorella sorokiniana, Chlamydomonas reinhardtii, Haematococcus pluvialis, Pleurochrysis carterae, and Phaeodactylum tricornutum (Lu and Xu, 2015; Park et al., 2014; Piotrowska-Niczyporuk and Bajguz, 2014).

Light is one of the key regulators of the cell cycle and interacts with plant hormones. Both light intensity and the cycle of alternating consecutive periods of light and darkness, i.e. photoperiod, are important. The quantity and quality of light is reflected in the amount of energy available for photosynthesis. Alternating cycles of light and darkness reflect natural environmental conditions of algal growth (Krzemińska et al., 2014, 2015; Strik et al., 2014).

Parachlorella kessleri (Chlorophyceae) is a unicellular freshwater green alga classified in the class Trebouxiophyceae. P. kessleri is characterized by a high growth rate and high biomass, starch, and lipid productivity. Moreover, it exhibits good tolerance to high temperature and a low tendency to form aggregates (Fernandes et al., 2013). These traits indicate that the species can be used in industrial productions of biomass.

The aim of the study was to determine the cumulative effect of addition of indole-3-acetic acid (IAA) and two light regimes: 16/8 h (light/dark) and continuous illumination on the growth parameters of Parachlorella kessleri.

\section{MATERIALS AND METHODS}

Parachlorella kessleri was obtained from the Culture Collection of Autotrophic Organisms (CCALA) from Dukelská in Czech Republic. The axenic cultures of $P$. kessleri were cultured in Bold Basal medium. The experiments were done in $300 \mathrm{ml}$ sterile Erlenmeyer flasks with $150 \mathrm{ml}$ of the medium with orbital shaking at 100 r.p.m.; the growth temperature was $26 \pm 1^{\circ} \mathrm{C}$. The microalgal cells were grown in the batch culture for 14 days.

Stock solutions of the indole-3-acetic acid phytohormone (IAA - Sigma-Aldrich) were prepared in $98 \%$ EtOH. An ethanolic solution of IAA was added to the BBM medium in an amount to provide a final concentration of $10^{-4} \mathrm{M}$ and a final concentration of $10^{-5} \mathrm{M}$ in the second variant. Control samples did not contain IAA. All tested samples were inoculated with the same amount of sterile cell suspension of $P$. kessleri pre-culture. The initial optical densities $\left(\mathrm{OD}_{650}\right)$ for the cultures were as follows: for the cultures growing under the photoperiod: 0.22 for the control: 0.20 for the concentration of $10^{-4} \mathrm{M}$ IAA, 0.20 for the concentration of $10^{-5} \mathrm{M}$ IAA, and under continuous illumination: 0.17 for the control, 0.15 for the concentration of $10^{-4} \mathrm{M}$ IAA and 0.16 for the concentration of $10^{-5} \mathrm{M}$ IAA.

The experimental variants included cultivation of $P$. kessleri at constant 24-h illumination (experimental variant I) and at a 16/18 h light/dark cycle (variant II) under light intensity of $80 \mu \mathrm{mol}$ photon $\mathrm{m}^{-2} \mathrm{~s}^{-1}$. Each culture variant was performed in three biological replicates.

The growth of the P. kessleri culture was monitored daily for 14 days by spectrophotometric measurements of the optical density at $650 \mathrm{~nm}\left(\mathrm{OD}_{650}\right)$ using a Cary $300 /$ Biomelt spectrophotometer. Based on the measurement of the optical density of the samples, growth parameters such as the specific growth rate and biomass doubling time were determined.

The specific growth rate $(\mu)\left(\right.$ day $\left.^{-1}\right)$ of $P$. kessleri microalgae was determined based on $\mathrm{OD}_{650}$ using the following formula:

$$
\mu=\frac{\ln N_{2}-\ln N_{1}}{t_{2}-t_{1}},
$$

where: $N_{1}$ is the initial biomass concentration at time $t_{1}$ and $N_{2}$ is the biomass concentration at time $t_{2}$ (Grudziński et al., 2016).

The biomass doubling time $T_{d}(\mathrm{~h})$ of $P$. kessleri microalgae was determined based on the specific growth rate $(\mu)$ using the following formula (Piasecka et al., 2014):

$$
T_{d}=\left(\frac{\ln 2}{\mu}\right) 24 .
$$

The number of cells was determined based on direct counting in the Burker chamber under the OLYMPUS/ CKX41SF microscope.

For dry weight determination, the known volume of culture suspension was filtered through pre-weighed and pre-dried filters (47 $\mathrm{mm}$ glass fibre paper filter Whatman $\mathrm{GF} / \mathrm{C})$. Next, the filters were dried in a laboratory drier Venticell/VC111 at $100^{\circ} \mathrm{C}$ for $24 \mathrm{~h}$ to constant weight. The difference in the weight of the filters was converted to 11 of a cell culture giving the content of dry cell weight in 11 of $P$. kessleri culture.

Statistical analysis of the results was carried out on the basis of statistical calculations in the Statistica (version 12.5, StatSoft Inc., USA). Differences in the measured parameters were compared using one-way ANOVA followed by a Tukey HSD test.

\section{RESULTS}

The growth of $P$. kessleri culture differed and depended on the applied illumination and concentrations of the added indole-3-acetic acid (IAA) phytohormone from the auxin group. 

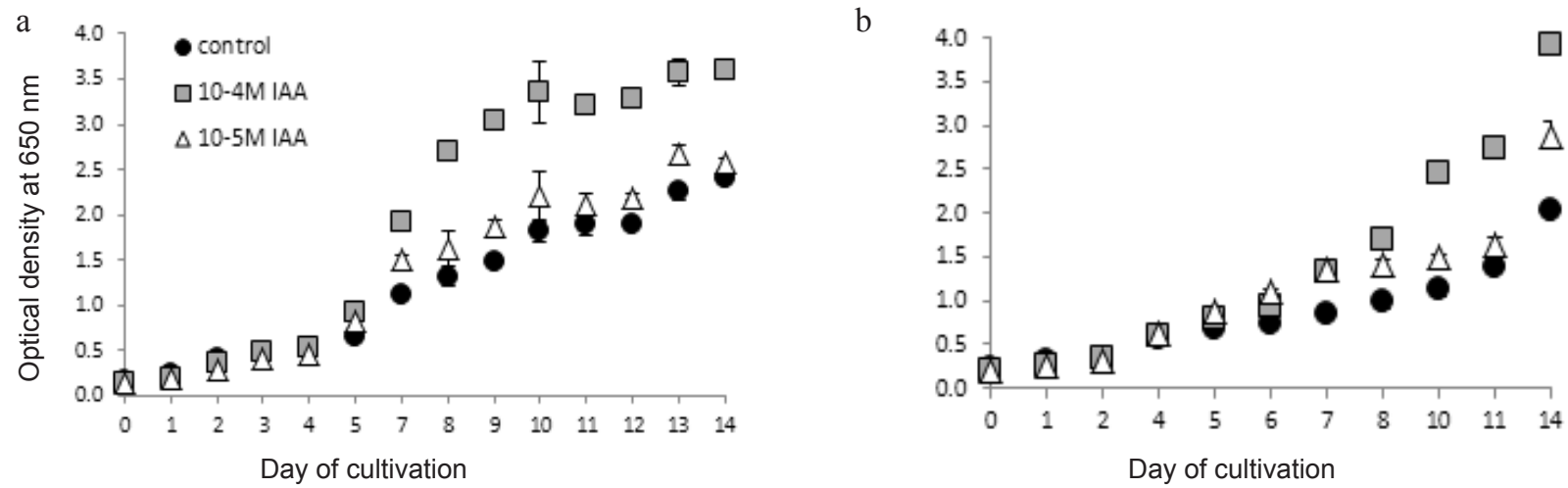

Fig. 1. Comparison of the growth of $P$. kessleri under the influence of IAA in: $\mathrm{a}$ - continuous illumination and $\mathrm{b}-$ photoperiod.

T a b l e 1. The impact of the use of the photoperiod, continuous illumination, and the addition of IAA on growth parameters: specific growth rate and biomass doubling time of $P$. kessleri

\begin{tabular}{ccc}
$\begin{array}{c}\text { Final } \\
\text { concentration } \\
\text { of IAA } \\
\left(\mathrm{mol} \mathrm{dm}^{-3}\right)\end{array}$ & $\begin{array}{c}\text { Specific } \\
\text { growth rate } \mu \\
\left(\text { day }^{-1}\right) \\
(0-8 \text { days })\end{array}$ & $\begin{array}{c}\text { Biomass } \\
\text { doubling time } \\
(\mathrm{h}) \\
(0-8 \text { days })\end{array}$ \\
\hline Continuous illumination & \\
$10^{-4}$ & $0.37 \pm 0.02 \mathrm{a}$ & $44.95 \pm 2.06 \mathrm{a}$ \\
$10^{-5}$ & $0.30 \pm 0.01 \mathrm{~b}$ & $55.57 \pm 2.72 \mathrm{~b}$ \\
Control & $0.25 \pm 0.01 \mathrm{c}$ & $65.35 \pm 2.46 \mathrm{c}$ \\
& Photoperiod & \\
$10^{-4}$ & $0.27 \pm 0.02 \mathrm{a}$ & $61.52 \pm 3.72 \mathrm{a}$ \\
$10^{-5}$ & $0.24 \pm 0.01 \mathrm{~b}$ & $66.31 \pm 1.52 \mathrm{~b}$ \\
Control & $0.18 \pm 0.01 \mathrm{c}$ & $93.88 \pm 2.73 \mathrm{c}$ \\
\hline
\end{tabular}

Different letters in columns indicate values significantly different $* \mathrm{p}<0.01$.

The comparison of the growth curve of $P$. kessleri is presented in Fig. 1. P. kessleri exhibited the lag growth phase between days 0-4 and next the linear phase was observed under continuous illumination and without IAA addition (control conditions) (Fig. 1a). The comparison of the light conditions applied revealed that the use of continuous illumination led to an increase in the values of $\mathrm{OD}_{650}$ relative to the cultures growing under the photoperiod conditions. In the control conditions and at the photoperiod applied, P. kessleri cells were in the linear growth phase (Fig. 1b).

The addition of the IAA phytohormone had an impact on the duration of the phases of growth curves of $P$. kessleri. Under the $16 / 8 \mathrm{~h}$ light/dark photoperiod, the stationary phase was not observed regardless of the amount of the added phytohormone. Changes in the shape of the growth curve are visible after day 6 of the culture, where the addition of $10^{-4} \mathrm{M}$ IAA had a stimulatory effect on growth, and the cells of $P$. kessleri entered the exponential phase earlier than the cultures containing a lower concentration of the phytohormone ( $10^{-5} \mathrm{M}$ IAA, Fig. $\left.1 \mathrm{~b}\right)$.

Under continuous illumination and at the higher concentration of the phytohormone $\left(10^{-4} \mathrm{M}\right.$ IAA), the cells of $P$. kessleri exhibited the exponential phase on days 5-10 and, next, the stationary phase was observed (Fig. 1a). The shape of the growth curve of the $P$. kessleri cultures supplemented with the lower concentration of IAA under continuous illumination was similar to the course of the growth curve in the cultures without the phytohormone addition. The $\mathrm{OD}_{650}$ values were higher in cultures containing the higher concentrations of the phytohormone $\left(10^{-4} \mathrm{M}\right.$ IAA) than in those containing the lower concentration of the phytohormone $\left(10^{-5} \mathrm{M}\right.$ IAA $)$.

The growth parameters of $P$. kessleri are summarized in Table 1. The indole-3-acetic acid (IAA) phytohormone has a stimulating effect on the growth of Parachlorella kessleri by increasing the specific growth rate and shortening the biomass doubling time relative to the control. The increase in the growth rate was proportional to the amount of the added phytohormone. A larger increase was observed upon addition of the higher concentrations of the phytohormone $-10^{-4} \mathrm{M}$ IAA than the lower concentrations $-10^{-5} \mathrm{M}$ IAA. At the 16/8 $\mathrm{h}$ light/dark photoperiod, the biomass doubling time was longer and the specific growth rate was lower than in cultures under continuous illumination. The highest growth rate $\left(\mu=0.37\right.$ day $\left.^{-1}\right)$ and the shortest doubling time $(44.95 \mathrm{~h})$ were observed under continuous illumination and the $10^{-4} \mathrm{M}$ IAA concentration (Table 1).

The addition of the phytohormone also resulted in an increase in the cell number in the P. kessleri culture, compared to the control (Fig. 2). At cultivation under continous illumination, the number of $P$. kessleri cells in the control culture increased from $7.1010^{8}$ to $1.2910^{10}$ cells during 14 days of the culture. The highest growth of the cell number, i.e. from $6.3810^{8}$ to $1.9310^{10}$ cells, was observed in the culture containing $10^{-4} \mathrm{M}$ IAA. For the $10^{-5} \mathrm{M}$ concentration of IAA, a small increase in the cell number was observed, compared to the control, i.e. from $6.8310^{8}$ to $1.3010^{10}$ cells 


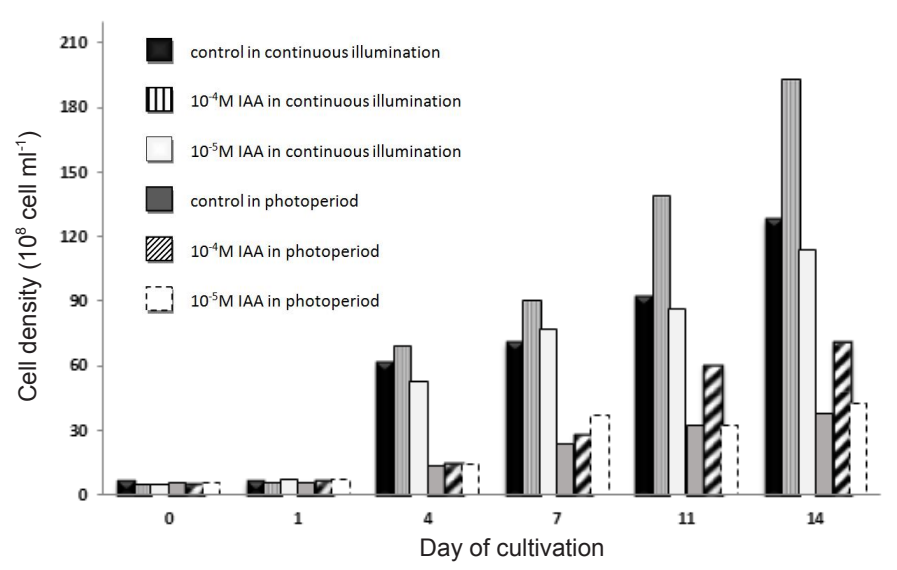

Fig. 2. Changes in the $P$. kessleri cell number induced by the addition of IAA under continuous illumination and the photoperiod.

over 1-14 days of cultivation. In the photoperiod cultivation, a decrease in the cell number was observed, compared to the culture under continuous illumination. The control cultivation of $P$. kessleri contained $5.9610^{8}$ cells on day 1 of cultivation and $3.7610^{9}$ cells on day 14 . A slightly higher number of cells were noted in the culture with $10^{-5} \mathrm{M}$ of IAA, i.e. from $7.2110^{8}$ to $4.2210^{9}$ cells over $1-14$ days of cultivation. The largest number of cells was found in the culture supplemented with $10^{-4} \mathrm{M}$ IAA, i.e. $7.0510^{8}$ cells on day 1 and $7.0910^{9}$ cells on day 14 of cultivation. However, compared to the culture in continuous illumination, the final number of cells grown in the photoperiod culture for 14 days was approximately 10 -fold lower. The varied biomass productivity was dependent on the concentrations of IAA used (Fig. 3). The addition of the IAA phytohormone resulted in an increase in the production of $P$. kessleri biomass. This relationship was observed at both the higher concentration of the phytohormone $10^{-4} \mathrm{M}$ IAA and the lower concentration $10^{-5} \mathrm{M}$ IAA. A higher final concentration of biomass was noted in the culture supplemented with the higher concentration of IAA $\left(10^{-4} \mathrm{M}\right.$ IAA $)$, compared to the culture containing the lower concentration of IAA $-10^{-5} \mathrm{M}$.

\section{DISCUSSION}

Phytohormones are naturally occurring biostimulators identified in terrestrial plants, fungi, mosses. They are also found in some species of algae, but at lower concentrations (Kemal, 2002; Lau et al., 2009; Lu and Xu, 2015; 2009; Yue et al., 2014). There have been many available reports published over the years about the impact of phytohormones on the growth and development of terrestrial plants (Bari and Jones, 2009; Voesenek and Van der Veen,

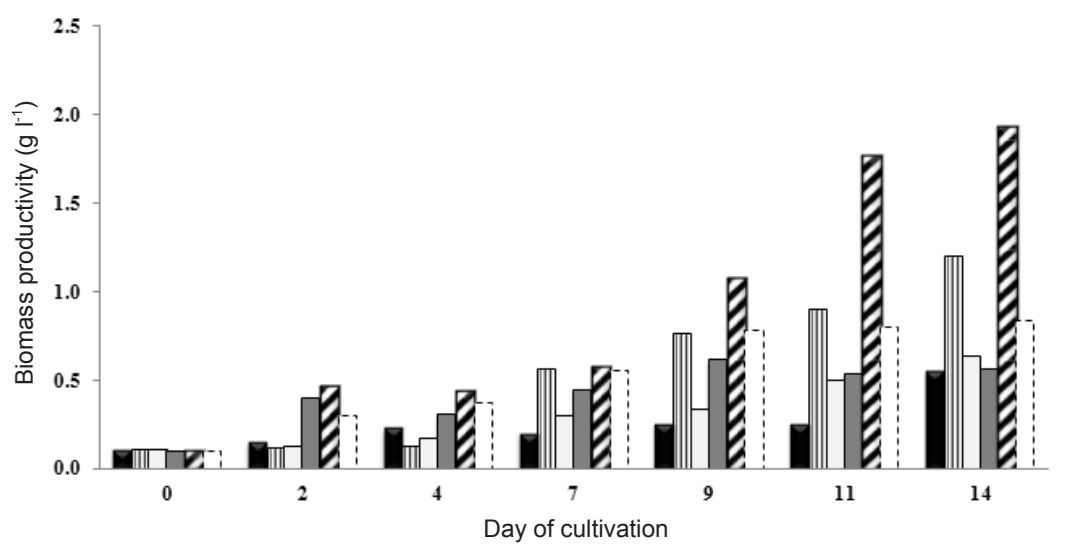

Fig. 3. The influence of the photoperiod, continuous illumination, and IAA addition on P. kessleri biomass productivity ( $\left.\mathrm{g} \mathrm{l}^{-1}\right)$. Legend as in Fig. 2. 
2014). However, the physiological, biochemical, and molecular roles of phytohormones in algae is not fully understood. In macroalgae, the role of phytohormones is probably similar to that in terrestrial plants. The effect of phytohormones in the case of microalgae is not so unequivocal (Mazur et al., 2001; Tarakhovskayaa et al., 2013). In terrestrial plants, IAA is responsible for growth promotion, cell division and enlargement, and tissue differentiation (Zhao, 2010). The possible role of IAA in microalgae is discussed. There are reports in the literature that exogenous IAA-supplementation of the cultivation medium may play a role in the stimulation of microalgal growth (Gonzales and Bashan, 2000). It is known that each species of algae reacts differently to the same phytohormone (Salama et al., 2014). However, not every concentration of phytohormones stimulates the growth of microalgae. Both too low and too high concentrations have a negative impact on the growth of algae. Therefore, it is highly important to find the optimal concentration of phytohormones for a particular species of algae. Physiological activities of IAA in relation to supporting cell division include response to light (Strik et al., 2014). So far, there have been no reports in the literature about the effect of IAA addition on the growth of P. kessleri. There are also no studies on the cumulative effect of the addition of IAA in various illumination conditions.

The indole-3-acetic acid (IAA) phytohormone increased the growth of the $P$. kessleri microalgae. As shown in Table 1, the maximum specific growth rate was found for the concentration of $10^{-4} \mathrm{M}$ IAA in continuous illumination $\left(0.37\right.$ day $\left.^{-1}\right)$, compared to the control $\left(0.25\right.$ day $\left.^{-1}\right)$, which corresponded to the shortest biomass doubling time (44.95 $\mathrm{h})$, compared to the control $(65.35 \mathrm{~h})$. In the same lighting conditions and the concentration of $10^{-5} \mathrm{M}$ IAA, the values of the specific growth rate were lower $\left(0.30\right.$ day $\left.^{-1}\right)$ and the biomass doubling time was longer $(55.57 \mathrm{~h})$ than for the concentration of $10^{-4} \mathrm{M}$ IAA. The literature shows that phytohormone supplementation, e.g. with indole-3-acetic acid and gibberellin (Lien et al., 1971; Vance, 1987), auxins and cytokinins (Czerpak et al., 1999), kinetin (6-furfurylaminopurine), significantly increases growth and cell division of Chlorella sp. (Li et al., 2007).

In turn, under the photoperiod $16 / 8 \mathrm{~h}$ (light/dark) (Table 1), the specific growth rate was lower and the biomass doubling time was longer, compared to the corresponding concentrations in continuous illumination. This correlation is observed at both concentrations of IAA (respectively for $10^{-4} \mathrm{M}$ IAA: 0.27 day $^{-1}$ and $61.52 \mathrm{~h} ; 10^{-5} \mathrm{M}$ IAA: 0.24 day $^{-1}$ and $66.31 \mathrm{~h}$ ), compared to the control $\left(0.18\right.$ day $^{-1}$ and $\left.93.88 \mathrm{~h}\right)$.

Liu et al. (2016) observed an improved growth rate in the cultures of Chlorella pyrenoidosa supplemented with IAA $\left(5.0-60 \mathrm{mg}^{-1}\right)$ in BG 11 medium with a light cycle $12 \mathrm{~h} / 12 \mathrm{~h}\left(100 \mu\right.$ mol photon $\left.\mathrm{m}^{-2} \mathrm{~s}^{-1}\right)$ than in the treatment without IAA. The growth rate of Scenedesmus quadricau$d a$ in the presence of IAA added in the range of $5.0-60 \mathrm{mg}$
$1^{-1}$ was lower and its maximum value was $0.64 \times 10^{-2}$ day $^{-1}$; in turn, it was $0.6510^{-2} \mathrm{day}^{-1}$ for the IAA non-supplemented culture (Liu et al., 2016). Piotrowska-Niczyporuk and Bajguz (2014) indicate that the growth of Chlorella vulgaris was stimulated at $0.1 \mu \mathrm{M}$ of IAA (under $50 \mu \mathrm{mol}$ photon $\mathrm{m}^{-2} \mathrm{~s}^{-1}$ light intensity and 16/8 $\mathrm{h}$ light/dark cycle); however, inhibitory effects were noted at a concentration of $100 \mu \mathrm{M}$ of IAA. Studies of exogenously added IAA $(100 \mu \mathrm{M})$ under continuous illumination $\left(80 \mu \mathrm{mol} \mathrm{m} \mathrm{m}^{-2} \mathrm{~s}^{-1}\right)$ showed no effect on the cell growth of $C$. vulgaris (Jusoh et al., 2015). These results indicate that the biological response to supplementation of auxin maybe species dependent.

Salama et al. (2014) reported that IAA addition enhanced Scenedesmus obliquus growth under continuous illumination of light at $45-50 \mu \mathrm{mol}$ photon $\mathrm{m}^{-2} \mathrm{~s}^{-1}$. Among the tested concentrations of $10^{-5}, 10^{-6}, 10^{-7}$, and $10^{-8} \mathrm{M}$, a maximum increase in Scenedesmus obliquus growth was observed at the concentration of $10^{-5} \mathrm{M}$ IAA. Microalgal growth increased with the increasing phytohormone concentrations. The maximum growth of $S$. obliquus, i.e. 17.7 $10^{6}$ cells ml ${ }^{-1}$, was observed on day 6 of cultivation. This constituted a 1.9-fold increase, compared to the control $\left(9.510^{6}\right.$ cells ml $\left.^{-1}\right)$. The present study has shown a 1.5 -fold higher number of $P$. kessleri cells in the case of $10^{-4} \mathrm{M}$ IAA in continuous illumination, compared to the control (1.93 $10^{10}$ cells $v s .1 .2910^{10}$ cells) during 14 days of cultivation (Fig. 2). At the concentration of $10^{-5} \mathrm{M}$ of IAA, a slight increase in the number of cells was observed, compared to the control $\left(1.3010^{10}\right.$ cells $)$. In the photoperiod cultivation, the greatest increase in the cell number was observed at the concentration of $10^{-4} \mathrm{M}$ IAA, compared to the control. For the concentration of $10^{-4} \mathrm{M}$ of IAA, a 1.9-fold higher number of $P$. kessleri cells was observed, compared to control $\left(7.0910^{9}\right.$ cells $v s .3 .7610^{9}$ cells $)$ and only a 1.12-fold increase in the cell number was noted at $10^{-5} \mathrm{M}$ of IAA (Fig. 2). This study has shown 3.4-fold higher total biomass productivity of $P$. kessleri at the addition of $10^{-4} \mathrm{M}$ IAA in the $16 / 8 \mathrm{~h} \mathrm{light/dark} \mathrm{photoperiod,} \mathrm{compared} \mathrm{to} \mathrm{the}$ control (1.93 vs. $\left.0.57 \mathrm{~g} \mathrm{l}^{-1}\right)$ during 14 culture days, and 2.2-fold higher biomass productivity of $P$. kessleri at the supplementation with $10^{-4} \mathrm{M}$ IAA in continuous illumination, compared to the control $\left(1.2 v s .0 .55 \mathrm{~g} \mathrm{l}^{-1}\right)$ (Fig. 3). The biomass productivity of $P$. kessleri obtained for the concentration of $10^{-5} \mathrm{M}$ IAA was 0.83 and $0.63 \mathrm{~g} \mathrm{l}^{-1}$, respectively, for the photoperiod and continuous illumination.

Park et al. (2014) examined the effect of various concentrations of IAA on biomass production in Chlamydomonas reinhardtii. They reported that the $c a .3 \mathrm{ppm}$ concentration of IAA was optimal for $C$. reinhardtii growth stimulation, as it yielded a $61 \%$ increase in biomass production, compared to the control with maximum biomass production $1.69 \mathrm{~g} \mathrm{l}^{-1}$. The most favorable conditions for the biomass production in $P$. kessleri were provided by the $16 / 8 \mathrm{~h}$ light/ dark photoperiod and the addition of $10^{-4} \mathrm{M}$ of IAA. 


\section{CONCLUSIONS}

1. The addition of indole-3-acetic acid increased biomass productivity of Parachlorella kessleri.

2. The higher concentration of indole-3-acetic acid $\left(10^{-4} \mathrm{M}\right)$ had a stimulating effect on the growth of Parachlorella kessleri microalgae in two variants of the lighting conditions applied.

3. Parachlorella kessleri show a maximum growth rate at continuous illumination and the concentration of $10^{-4} \mathrm{M}$ of indole-3-acetic acid.

4. The comparison of the culture conditions shows that continuous illumination and the concentration of $10^{-4} \mathrm{M}$ of indole-3-acetic acid promoted cell proliferation of Parachlorella kessleri more effectively than 16/8 h (light/ dark photoperiod).

Author contribution: E. Magierek performed the experiments, analysed and interpreted the data, drafted the manuscript, and approved the version of manuscript to be submitted. I. Krzemińska devised the entire set of the experiments, analysed and interpreted the data, drafted the manuscript, made a revision based on other authors' contributions, approved, and submitted the final version of the article. J. Tys analysed the data and approved the version of the manuscript to be submitted.

Conflict of interest: The authors declare that there is no conflict of interest about the publication of this work.

\section{REFERENCES}

Bari R. and Jones J.D.G., 2009. Role of plant hormones in plant defence responses. Plant Molecular Biol., March, 69, 473-488

Czerpak R., Krotke A., and Mical A., 1999. Comparison of stimulatory effect of auxins and cytokinins on protein, saccharides and chlorophyllssal content in Chlorella pyrenoidosa Chick. Pol Arch Hydrobiol., 46: 71-82.

Fernandes B., Teixeira J., Dragone G.,Vicente A.A., Kawano S., Bišova K., Př̌ibyl P., Zachleder V., and Vitova M., 2013. Relationship between starch and lipid accumulation induced by nutrient depletion and replenishment in the microalga Parachlorella kessleri. Biores. Technol., 144, 268-274.

Gonzalez L.E. and Bashan Y., 2000. Increased Growth of the Microalga Chlorella vulgaris when coimmobilized and cocultured in alginate Beads with the plant-growth-promoting bacterium Azospirillum brasilense. Appl. Environ. Microbiol., 66, 1527-1531.

Grudziński W., Krzemińska I., Luchowski R., Nosalewicz A., and Gruszecki W.I., 2016. Strong-light-induced yellowing of green microalgae Chlorella: A study on molecular mechanisms of the acclimation response. Algal Research, 16, $245-254$
Jusoh M., Loh S.H., Chuah T.S., Aziz A., and Cha T.S., 2015. Indole-3-acetic acid (IAA) induced changes in oil content, fatty acid profiles and expression of four fatty acid biosynthetic genes in Chlorella vulgaris at early stationary growth phase. Phytochemistry, 111, 65-71.

Kemal M., 2002. Auxin (Indole-3-acetic acid), Gibberellic acid (GA3), Abscisic Acid (ABA) and Cytokinin (Zeatin) production by some species of mosses and lichensm. Turk. J. Bot., 26, 13-18.

Krzemińska I., Pawlik-Skowrońska B., Trzcińska M., and Tys J., 2014. Influence of photoperiods on the growth rate and biomass productivity of green microalgae. Bioprocess Biosyst. Eng., 37, 735-741.

Krzemińska I., Piasecka A., Nosalewicz A., Simionato D., and Wawrzykowski J., 2015. Alterations of the lipid content and fatty acid profile of Chlorella protothecoides under different light intensities. Biores. Technol., 196, 72-77.

Lau S., Shao N., Bock R., Jurgens G., and De Smet I., 2009. Auxin signaling in algal lineages: fact or myth? Trends Plant, 14: 182-188.

Li T., Wang C., and Miao J., 2007. Identification and quantification of indole-3-acetic acid in the kelp Laminaria japonica Areschoug and its effect on growth of marine microalgae. J. Appl. Phycol., 19, 479-484.

Lien T., Pettersen R., and Knutsen G., 1971. Effects of indole3 -acetic acid and gibberellin on synchronous cultures of Chlorella fusca. Physiol Plant, 24, 185-190.

Liu J., Qiu W., and Song Y., 2016. Stimulatory effect of auxins on the growth and lipid productivity of Chlorella pyrenoidosa and Scenedesmus quadricauda. Algal Res., 18, 273-280.

Lu Y. and Xu J., 2015. Phytohormones in microalgae: a new opportunity for microalgal biotechnology? Trends in Plant Sci., 20, 273-82.

Markou G. and Nerantzis E., 2013. Microalgae for high-value and biofuels production: A review. Biotechnol. Advances, 31, 1532-1542.

Mata T., Martins A., and Caetano N., 2010. Microalgae for biodiesel production and other applications. A review. Renewable Sustainable Energy Reviews, 14, 217-232.

Mazur H., Konop A., and Synak R., 2001. Indole-3-acetic acid in the culture medium of two axenic green microalgae. J. Appl. Phycol., 13, 35-42.

Park W.K., Yoo G., Moon M., Kim C.W., Choi Y.E., and Yang J.W., 2014. Phytohormone supplementation significantly increases growth of Chlamydomonas reinhardtii cultivated for biodiesel production. Appl. Biochem. Biotechnol., 171, 1128-1142.

Piasecka A., Krzemińska I., and Tys J., 2014. Physical methods of microalgal biomass pretreatment. Int. Agrophys., 28, 341-348.

Piotrowska-Niczyporuk A. and Bajguz A., 2014. The effect of natural and synthetic auxins on the growth, metabolite content and antioxidant response of green alga Chlorella vulgaris (Trebouxiophyceae). Plant Growth Regul., 73, 57-66. 
Salama E.S., Kabra A.N., Ji M.K., Kim J.R., Min B., and Jeon B.H., 2014. Enhancement of microalgae growth and fatty acid content under the influence of phytohormones. Biores. Technol., 172, 97-103.

Stirk W.A., Bálint P., Tarkowská D., Novák O., Maróti G., Ljung K., Turečková V., Strnad M., Ordög V., and van Staden J., 2014. Effect of light on growth and endogenous hormones in Chlorella minutissima (Trebouxiophyceae). Plant Physiol. Biochem., 79, 66-76.

Tarakhovskayaa E.R., Kangb E.J., and Kimb K.Y., 2013. Influence of phytohormones on morphology and chloro- phyll a fluorescence parameters in embryos of Fucus vesiculosus L. (Phaeophyceae). Garbaryc Russian J. Plant Physiol., 60, 176-183.

Vance B.D., 1987. Phytohormone effects on cell division in Chlorella pyrenoidosa Chick (TX-7-11-05)(Chlorellaceae). J. Plant Growth Regul., 5, 169-173.

Voesenek A.C.J. and Van der Veen R., 2014. The role of phytohormones in plant stress: too much or too little water. Acta Botanica Neerlandica, 43, 91-127.

Yue J., Hu X., and Huang J., 2014. Origin of plant auxin biosynthesis. Trends Plant Sci., 19, 764-770.

Zhao Y., 2010. Auxin biosynthesis and its role in plant development. Annu. Rev. Plant. Biol., 61, 49-64. 